\title{
Transformación de un auto Sedan (volkswagen) a híbrido y totalmente eléctrico
}

\section{Transformation of a Sedan car (volkswagen) to hybrid and fully electric}

\author{
CHAVEZ-MENDOZA, Juan Manuel ${ }^{* \dagger}$ \\ Universidad Tecnológica Fidel Velázquez \\ ID $1^{\text {er }}$ Autor: Juan Manuel, Chavez-Mendoza
}

DOI: 10.35429/JID.2020.11.4.19.25

Recibido 23 de Junio, 2020; Aceptado 12 de Diciembre, 2020

\begin{abstract}
Resumen
El este trabajo se hace una propuesta de transformación del vehículo vw sedán a hibrido y eléctrico como una alternativa viable, para reducir las emisiones contaminantes por parte de los vehículos de combustión y a los elevados costos que los combustibles convencionales han logrado alcanzar en los últimos tiempo, se han comenzado a implementar nuevas estructuras que permitan mejorar las condiciones de propulsión del vehículo reduciendo los contaminantes. Por este motivo es que resulta atractiva la opción de vehículos que contienen dos sistemas para generar su funcionamiento la opción que vamos a analizar es de vehículo híbrido con un arreglo en paralelo, en el cual la energía eléctrica que lo impulsa proviene de baterías y alternativamente de un motor de combustión interna que mueve un generador. Normalmente, un motor de combustión interna también puede impulsar las ruedas en forma directa. En el diseño de un automóvil híbrido, el motor térmico es la fuente de energía que se utiliza como última opción, y se dispone un sistema electrónico para determinar qué motor usar y cuándo hacerlo
\end{abstract}

Conversión, Energía eléctrica, Contaminación

\begin{abstract}
In this work, a proposal is made to transform the vw sedan vehicle into a hybrid and electric vehicle as a viable alternative, to reduce polluting emissions from combustion vehicles and the high costs that conventional fuels have managed to achieve in recent times, New structures have been implemented to improve vehicle propulsion conditions by reducing pollutants. For this reason, the option of vehicles that contain two systems to generate their operation is attractive. The option that we are going to analyze is a hybrid vehicle with a parallel arrangement, in which the electrical energy that drives it comes from batteries and alternatively from an internal combustion engine that drives a generator. Typically, an internal combustion engine can also drive the wheels directly. In the design of a hybrid car, the heat engine is the energy source that is used as last option, and an electronic system is arranged to determine which engine to use and when to use it.
\end{abstract}

\section{Conversion, Electrical energy, Pollution}

Citación: CHAVEZ-MENDOZA, Juan Manuel. Transformación de un auto Sedan (volkswagen) a híbrido y totalmente eléctrico. Revista del Diseño Innovativo. 2020, 4.11: 19-25

\footnotetext{
*Correspondencia al Autor (Correo electrónico: jmanuel.chavez@utfv.edu.mx)

$\dagger$ Investigador contribuyendo como primer autor.
} 


\section{Introducción}

La implementación de este trabajo es de establecer una solución a la necesidad de crear nuevos vehículos que tengan menos emisiones contaminantes en nuestro caso escogimos el vehículo sedan Volkswagen "vocho", donde se realizaron cálculos e investigación para la conversión de vehículo eléctrico e hibrido, sabiendo que el inicio de este vehículo es en Alemania. En el año 1933, Ferdinand Porsche se reunió con Adolf Hitler para discutir la idea Del Volkswagen. Hitler quería un vehículo que fuese capaz de transportar a 5 personas a 100 $\mathrm{km} / \mathrm{h}$ y que costara solamente 1000 Marcos en ese entonces.

Esta era la oportunidad de Ferdinand Porsche para llevar su idea a cabo y al mismo tiempo ayudar a Hitler para entregarle a los Volkswagen. Nuestra tarea siendo ingeniero está en la evolución del auto, donde se a presentado a los vehículos eléctricos e hibrido, esta idea fue inicio desde 1938 cuando Robert Davidson consiguió mover una locomotora a 6 $\mathrm{km} / \mathrm{h}$ sin usar carbón ni vapor. El coche eléctrico triunfa por su simplicidad, fiabilidad, suavidad de marcha, costos de energía,

Empezando este trabajo se analizó las principales características de los autos eléctricos e híbridos se encontró que la clasificación depende de tipo de corriente que maneja (continua/ alterna), en la posición del motor (series, combinado. paralelo) la baterías también es un dato muy importante ya que es el corazón del sistema y el elemento más costoso y pesado. La batería no sólo almacena energía eléctrica para utilizarla en el vehículo, sino que también determina la potencia que puede usar el motor, la autonomía y el diseño del vehículo.

Encontramos un motor compatible (impulse 9 ev dc). Se trata de motor de diámetro de 9,25, de corriente continua con un eje de doble extremo y está pre-perforado para permitir el avance del cepillo. Ya demostrando todos los datos técnicos se entregará toda la memoria de cálculo así tener un argumento teórico para futuras generaciones.

\section{Historia Volkswagen Sedan}

Literalmente el significado de la palabra "Volkswagen" es "el auto del pueblo". En Alemania, la idea no era exactamente nueva. Antes de los años treinta se hicieron muchos esfuerzos para crear automóviles más simples que estuviesen al alcance de todos pero ninguno lo hizo como el Volkswagen. Casi todos los automóviles antes de los años 30 aun cuando intentaron satisfacer las más simples necesidades de los trabajadores sobrepasaban ampliamente la capacidad de endeudamiento de estos.

Después de la segunda guerra mundial se comenzó a vislumbrar un fenómeno en el cual los dueños de automóviles los modificaban, esto surgió en algunos casos por la necesidad de contar con elementos que los distinguieran de los que se producían en serie. Al inicio se hacían pequeños cambios que con el tiempo se convirtieron en modificaciones estructurales que sorprendían a propios y extraños. El automóvil pasó de ser un medio de transporte a un lujo que proyecta la personalidad de los conductores que cada día se preocupan más por los accesorios de su vehículo.

Fue tanta la demanda del fenómeno que se establecieron empresas dedicadas a proveer de accesorios de todo tipo para modificar los vehículos se denominan auto boutique, son las encargadas de modificar los componentes de los autos e incluso en su estructura. Las personas que se dedican a este negocio conocen de mecánica, electrónica e ingeniería motriz para garantizar que los resultados sean los más convincentes (VOLKSWAGEN, s.f.)

\section{El vehículo eléctrico}

El vapor no conseguía sustituir al coche de caballos, le salió un competidor nuevo. Tras los primeros experimentos de Jedlik (1828, en imagen) y Thomas Davenport (1835), encontramos el primer vehículo eléctrico en 1838, cuando Robert Davidson consiguió mover una locomotora a $6 \mathrm{~km} / \mathrm{h}$ sin usar carbón ni vapor. Entre 1832 y 1839 Robert Anderson inventó el primer carruaje de tracción eléctrica, con pila de energía no recargable. 
Poco después se patentó la línea electrificada, pero eso no valía para coches, solo para trolebuses o trenes. Las primeras baterías recargables aparecieron antes de 1880 , ahí comenzaron realmente.

Se hicieron con las carreteras en poco tiempo, en 1900 podemos considerar su apogeo, cuando eran los coches que más se vendían, mucho más que los de vapor o gasolina. De hecho, en 1899 un coche eléctrico, "La Jamais Contente", superó por primera vez los $100 \mathrm{~km} / \mathrm{h}$ e instauró un récord de velocidad. Los primeros coches de gasolina eran muy contaminantes, sucios, ruidosos, requerían bencina o gasolina (no era fácil comprarla al principio), había que cambiar de marcha muy rudimentariamente, había que arrancarlos con manivela y en cualquier lugar eran susceptibles de fallar.

Eso acabó cambiando... un poco. El coche eléctrico triunfaba por su simplicidad, fiabilidad, suavidad de marcha, sin cambio de marchas ni manivela, no hacían ruido, eran veloces, la autonomía era razonable y su coste era soportable para la burguesía y las clases altas, los primeros usuarios de automóviles. Superaban por 10 a 1 a los de gasolina. Sin embargo el coche de gasolina recibió el motor de arranque (1912), Henry Ford inventó la producción en masa mecanizada, la gasolina alcanzó un precio muy popular, se empezaron a abrir carreteras al tráfico y entonces la autonomía pasó a ser una característica muy valorada, además del precio. (COSTAS, MOTORPASION, 2010)

\section{El vehículo eléctrico}

Hoy en día el vehículo eléctrico representa el $3 \%$ del total del parque vehicular mundial y de acuerdo al Navigant Research, ese porcentaje incrementará a un 7\% para 2020 (alrededor de 6.6 millones de autos al año).

Se puede pensar que el auto eléctrico es una innovación reciente que se está abriendo paso en el mercado. Sin embargo, siendo estrictos el auto eléctrico no es precisamente una novedad. De hecho, los primero modelos salieron a las calles durante la primera mitad del siglo XIX y fueron tan populares que para 1900 ya representaban un tercio de los autos de la época. El auto de combustión lo reemplazó por su menor costo y terminó casi en el olvido.
Hoy está de vuelta y para quedarse. Sus características y cualidades se ajustan muy bien a nuestra actualidad donde la economía y el medio ambiente demandan alternativas. La clave está en su ingeniería, pero ¿cómo funciona?

Todo se basa en motores eléctricos que convierten la electricidad en energía mecánica para mover las ruedas. Su funcionamiento es relativamente sencillo, al menos comparado con los de combustión. Pero te lo explicamos un poco más a detalle. Los elementos más característicos de los vehículos eléctricos son el puerto de carga, transformador, batería, controlador y el motor. (AGUILAR, 2015)

\section{Puerto de carga}

El puerto de carga de un auto eléctrico es el punto por el que recargamos el auto. Generalmente es una toma exterior al estilo de la toma de gasolina de los autos convencionales. Cada marca fabrica su propio tipo de puertos pero usualmente son contactos trifásicos. Para dejarlo más claro, es por donde enchufas el auto a la electricidad. Tal cual, es el puerto de entrada de la energía a nuestra unidad.

\section{Transformador}

Son los que se encargan de transformar la intensidad y el voltaje de la corriente eléctrica en valores válidos para el funcionamiento del automóvil. También ayudan a la refrigeración evitando derrames y accidentes.

\section{Controlador}

Los controladores se encargan de que el funcionamiento sea óptimo, ya que recibe y recarga la energía del motor. Al necesitar pocos elementos para funcionar, el auto eléctrico, se vuelve mucho más eficiente que un coche de combustión, ya que pueden convertir hasta el $60 \%$ de su energía en energía mecánica; un auto de combustión interna que sólo llega a convertir hasta el $20 \%$.

Las opciones son: motor con escobillas de corriente continua, motores de inducción y varias más. Dentro de cada una de estas opciones hay otras, por ejemplo, en motores con escobillas CC, puede tener campo bobinado o con imanes permanentes. 
Esto también es válido para un motor sin escobillas, aunque cuando se está buscando opciones más económicas, los motores de corriente continua son los que tienen menor precio. Actualmente solo recomendamos sistemas con escobillas en el caso de proyectos de uso personal donde se cuente con escaso presupuesto y la eficiencia no sea el factor más importante. (AGUILAR, 2015)

\section{Tipo de corriente}

Ahora cuando se trata de proyectos comerciales o donde sea necesarias características como la alta eficiencia, el mantenimiento cero y la confiabilidad de funcionamiento, sin dudas los motores AC son los adecuados. Actualmente un buen motor AC y su Controlador de velocidad (Invertir) con capacidad de frenado regenerativo solo salen unos pocos dólares más que los antiguos sistemas DC. (Barrios, 2014) DC (del inglés Direct Current - Corriente Continua)

En los autos que tienen un motor eléctrico de corriente continua, esta batería iría directamente conectada al motor. En cambio, en los coches eléctricos que tienen un motor eléctrico de corriente alterna, la batería va conectada a un inversor. Las baterías de Litioion almacenan la energía que le cede el cargador en forma de corriente continua (DC). Esta batería principal es el medio por el que se alimenta todo el coche eléctrico. Este tipo de motor es más tradicional, la aceleración está más lenta, pero tiene una velocidad máxima muy alta. Además los motores de corriente continua son menos caros que los motores de corriente alterna.

AC (del inglés Alternating Current - Corriente Alterna)

En los coches eléctricos que tienen un motor eléctrico de corriente alterna, la batería va conectada a un inversor. Los motores eléctricos de corriente alterna tienen buena fama ya que se utilizan en los coches de TESLA y Nissan, entre otras empresas. Son más caros que los motores de corriente continua, pero tienen una tasa de aceleración rápida y son compatibles con los sistemas de frenado regenerativo. Este sistema convierte el impulso del motor eléctrico y utilízalo para recargar las baterías. (Electricocar, 2013)

\section{Características de los vehículos híbridos}

Un híbrido combina dos motorizaciones, un motor de combustión interna y otro eléctrico alimentado por baterías adicionales a la principal. Son una realidad desde hace muchos años y poco a poco empiezan a hacerse populares por la crisis, alza de combustibles, abaratamiento de la tecnología y concienciación ambiental.

\section{Clasificación}

- Híbrido en serie: El motor de combustión interna (en adelante motor térmico) no tiene conexión mecánica con las ruedas, sólo se usa para generar electricidad. Dicho motor funciona a un régimen óptimo y recarga la batería hasta que se llena, momento en el cual se desconecta temporalmente. La tracción es siempre eléctrica.

- Híbrido en paralelo: Tanto el motor térmico como el eléctrico se utilizan para dar fuerza a la transmisión a la vez. Es una solución relativamente sencilla, pero no es la más eficiente.

- Híbrido combinado: Cualquier combinación de los dos motores sirve para impulsar al coche, es como un híbrido en serie pero con conexión mecánica a las ruedas. Es una solución muy eficiente pero mucho más compleja a nivel mecánico y electrónico.

- $\quad$ (COSTAS, MOTORPASION, 2009)

El sistema más eficiente es en serie, y el que menos el paralelo. Existe otra forma de clasificar a los híbridos:

Micro híbrido: En las paradas se apaga el motor térmico. Cuando se quiere reanudar la marcha un alternador reversible arranca el motor utilizando energía recuperada previamente a la detención. Sólo ahorra en ciclo urbano y no hay un motor eléctrico que impulse al coche. 
- Semihíbrido o mild-hybrid: El motor eléctrico se utiliza como una asistencia al motor térmico y además es generador de energía en las frenadas y retenciones, pero no puede impulsarse de forma $100 \%$ eléctrica (motor térmico apagado) aunque sí con el motor térmico sin consumir pero moviendo sus piezas mecánicas.

- Híbrido puro o full-hybrid: Se puede circular en determinadas condiciones sólo con el motor eléctrico, mientras el térmico está totalmente apagado y no mueve sus piezas. Este cambio puede ser de forma automática o voluntaria.

- $\quad$ Híbrido enchufable o PHEV: Pertenece a este grupo si sus baterías son recargables mediante energía eléctrica convencional, es decir, enchufándolo, y recorre al menos 32 kilómetros sin necesidad de otro sistema de propulsión. Coche eléctrico de rango extendido o EREV: Como el caso anterior, pero si además es un híbrido en serie. En la práctica, se les considera coches eléctricos porque no necesitan el motor térmico más que para sostener la carga, y pueden funcionar sin ellos al $100 \%$. Esto significa que cuando se acaban las baterías el motor térmico se usa sólo para generar electricidad a un régimen constante para aumentar la autonomía a un coste por kilómetro bajísimo. (COSTAS, MOTORPASION, 2009)

\section{Memoria de cálculo}

Potencia necesaria para vencer la resistencia al avance:

$$
\begin{aligned}
& P w=(R t * v) /(75 * n t) \\
& P w=(215.0576 * 35.2777) /(75 * 0.85) \\
& P \mathrm{w}=119.0076 \mathrm{CV}
\end{aligned}
$$

Rt: resistencia al avance $(\mathrm{kg})$

v: velocidad del auto $(\mathrm{m} / \mathrm{s})$

nt: rendimiento de la transmisión

Calculo de la relación de la caja de cambios y sus velocidades

$R d=\frac{205 *(80.60 * 2)+25.4 * 15}{2}=313.5 \mathrm{~mm}$

$$
R f=\frac{n m \times 2 \pi x R d}{r 4 x v \max x 60}=\frac{4400 * 2 \pi * 0.3135}{88 * 35.2777 * 60}=4.653
$$

$\mathrm{Rd}$ : radio de la rueda en $\mathrm{m}$ nm: rpm del motor rf: relación del diferencial vmáx: velocidad máxima del auto en $\mathrm{m} / \mathrm{s}$ n2: rpm a potencia máxima n1: rpm a torque máximo r1, r2, r3 y r4: relación de transmisión de cada cambio

$$
\begin{aligned}
& r 4=\frac{n 2 \times 2 \pi \times R d}{r f x \operatorname{vmaxx60}}=\frac{4400 * 2 \pi * 0.3135}{4.653 * 35.2777 * 60}=0.880 \\
& r 3=r 4 x \frac{n 2}{n 1}=0.88 \frac{4400}{2600}=1.4892 \\
& r 2=r 3 x \frac{n 2}{n 1}=1.4892 \frac{4400}{2600}=2.52 \\
& r 1=r 2 x \frac{n 2}{n 1}=2.52 \frac{4400}{2600}=4.2 \\
& r m a=\frac{n 2 \times 2 \pi x R d}{r f x \operatorname{vmaxx} 60}=\frac{4400 * 2 \pi * 0.3135}{4.653 * 8.001 * 60}=3.88
\end{aligned}
$$

\section{Esfuerzo motriz}

$F=\frac{M m \times r i x r f \times n t}{R d}=\frac{103.986 * 2.6923 * 4.653 * 0.85}{0.3135}=3531.9351 \mathrm{~N}$

Mm: Torque en el motor

F: Esfuerzo motriz

$\mathrm{Mm}=10.6 \mathrm{~kg}-\mathrm{m}=103.986 \mathrm{~N}-\mathrm{m}$

Capacidad de aceleración

$$
\begin{aligned}
& \gamma=\frac{F-R t}{m e}=\frac{3531.9351-2109.715}{1212}=1.173 \\
& \mathrm{Me}=1.01 \times \mathrm{P}=1.01 * 1200=1212 \mathrm{~kg}
\end{aligned}
$$

Tiempo de aceleración 0 a $100 \mathrm{~km} / \mathrm{hr}$ $(27.77 \mathrm{~m} / \mathrm{seg})$

$$
\begin{aligned}
& t v f-v i=1 / \gamma \int_{v i}^{v f} d v \\
& t 0-27.77=1 / 1.1734 \int_{0}^{27.77} d v=23.6728 \mathrm{seg}
\end{aligned}
$$

\section{Calculo de potencia y torque del vehículo}

El motor cuenta con un Troque de $10.6 \mathrm{~kg}-\mathrm{m}$ a 2600 rpm y una potencia máxima de 55 hp @ $4400 \mathrm{rpm}$. Para convertir de caballos de fuerza a caballos de vapor se debe multiplicar por 0.985923 que es el Factor de conversión.

Es decir 55 hp x 0.985923 Fac. $=55.785 \mathrm{Cv}$ 


\section{Modificaciones a realizar para la conversión $100 \%$ eléctrico}

Motor eléctrico y baterías. Para el motor eléctrico se tiene el modelo IMPULSE $9 \mathrm{EV}$ DC Se trata de un "diámetro de 9,25, de corriente continua con un eje de doble extremo y está pre-perforado para permitir el avance del cepillo, ya sea en neutral, CWDE avanzado o CCWDE avanzada (estándar). Con las especificaciones del motor sabemos que es necesario un voltaje de $144 \mathrm{v}$ a $450 \mathrm{~A}$ cada batería individual maneja 24v A 18 entonces para compensar

Ventajas de la batería de Litio-Ion sobre las baterías convencionales de plomo-ácido

- $\quad$ Alta densidad de energía: más energía con menos peso;

Altas corrientes de carga (acorta el tiempo de carga

Altas corrientes de descarga (permite, por ejemplo, alimentar una cocina eléctrica con una bancada de baterías pequeña);

- $\quad$ Larga vida útil de la batería (hasta seis veces más que la de una batería convencional)

Alta eficiencia entre la carga y la descarga (muy poca pérdida de energía debido al calentamiento);

Mayor continuidad de la corriente disponible.

Especificaciones de la batería:

Baterías de Litio-Ion de 24V 180Ah 4,75kWh

Tecnología Fosfato de hierro y litio (LiFePo4)

Tensión nominal 26,4 V

Capacidad nominal $180 \mathrm{Ah}$

Potencia nominal 4,75 KWh

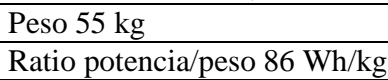

Dimensiones (al x an x p ) 625 x $195 \times 355 \mathrm{~mm}$

Tensión de corte de la carga a $0,05 \mathrm{C} 28,8 \mathrm{~V}$

Tensión de corte de descarga $20 \mathrm{~V}$

Corriente de carga/descarga

recomendada 54 A (0,3C)

Corriente máxima de carga (1C) $180 \mathrm{~A}$

Corriente máxima de descarga $(1,5 \mathrm{C}) 270 \mathrm{~A}$

Corriente de descarga por pulsación (10s) $1.000 \mathrm{~A}$

Cantidad de ciclos @ 80\% DOD (0,3C) 2000

Configuración de series Sí, hasta 2

(más series si se solicitan)

Configuración paralela Sí, fácilmente hasta 4

(más en paralelo si se solicita)

Temp. de trabajo para carga $0 \sim 45{ }^{\circ} \mathrm{C}$

Temp. de trabajo para descarga $-20 \sim 55^{\circ} \mathrm{C}$

Temp. de almacenamiento $-20 \sim 45^{\circ} \mathrm{C}$

Tabla 1 Ficha técnica de la batería (Compan,2016)

ISSN-2523-6830

ECORFAN $^{\circledR}$ Todos los derechos reservados

\section{Resultados}

Dadas las características del chasís del auto seleccionado, el motor eléctrico fue instalado directo a la transmisión mediante una placa de acoplamiento, y el paquete de baterías se dispuso en el piso del asiento trasero del propio auto, las cajas de conexiones y accesorios de control se distribuyeron en la cajuela frontal del auto. Todo el proceso de montaje y pruebas se desarrolló en las instalaciones y el vehículo quedó a resguardo de los patrocinadores para su disposición final.

\section{Referencias}

AGUILAR, C. A. (3 de MAYO de 2015). MOTORBIT. Recuperado el 2016, de http://motorbit.com/asi-funciona-un-motor-deauto-electrico/?pais=

Barrios, G. G. (2014). AUTO LIBRE. Recuperado el 2016, de http://autolibre.blogspot.com/2009/05/cual-esel-mejor-motor-para-un-auto.html

Compan, E. C. (2016). ELECTRIC CAR PARTS COMPANY. Obtenido de http://www.electriccarpartscompany.com/ImPul se-9-EV-DC-Motor-br-72-156V-400A-brDouble-ended-shaft--advanced-timing-1125DE-Shaft-1125-CE-Shaft_p_68.html

COSTAS, J. (18 de MAYO de 2009). MOTORPASION. Obtenido de http://www.motorpasion.com/coches-hibridosalternativos/que-es-un-coche-hibrido

COSTAS, J. (7 de MAYO de 2010). MOTORPASION. Recuperado el 2016, de http://www.motorpasion.com/coches-hibridosalternativos/historia-de-los-coches-electricos

Electricocar. (4 de NOVIEMBRE de 2013). ELECTRICOCAR. Recuperado el 2016, de http://www.electricocar.com/motores-paracoches-electricos.html

Energy, V. (s.f.). victronenergy bluepower. Obtenido de https://www.victronenergy.com/upload/docume nts/Datasheet-Lithium-ion-and-Lynx-ionES.pdf 
VOLKSWAGEN, H. D. (s.f.). Especificaciones por Año. Recuperado el 2016, de VW tiene el record:

http://oldvwgarage.com/vws/historia_del_voch o.htm\#Historia 